\title{
Resveratrol inhibits the hedgehog signaling pathway and epithelial-mesenchymal transition and suppresses gastric cancer invasion and metastasis
}

\author{
QIAN GAO $^{1}$, YUAN YUAN ${ }^{2}$, HUI-ZHONG GAN ${ }^{1}$ and QIONG PENG ${ }^{1}$ \\ ${ }^{1}$ Department of Gastroenterology, The Third Affiliated Hospital of Anhui Medical University, Hefei, Anhui 230061; \\ ${ }^{2}$ Central Laboratory of Binhu Hospital, The Third Affiliated Hospital of Anhui Medical University, \\ Hefei, Anhui 230601, P.R. China
}

Received April 13, 2014; Accepted January 22, 2015

DOI: $10.3892 / \mathrm{ol} .2015 .2988$

\begin{abstract}
The hedgehog (Hh) signaling pathway is vital to vertebrate development, the homeostatic process and tumorigenesis. Epithelial-mesenchymal transition (EMT) is a cellular process during which epithelial cells become mesenchymal-appearing cells, which in turn promotes cancer metastasis and invasion. Resveratrol is a natural polyphenolic compound found in grapes, a variety of berries, peanuts and other plants. Numerous studies have demonstrated that the Hh signaling pathway is able to regulate the EMT, and that resveratrol can suppress carcinoma invasion and metastasis. In addition, certain studies have indicated that resveratrol can inhibit the Hh signaling pathway and EMT in cancers other than gastric cancer. The purpose of the present study was to investigate the inhibitory effect of resveratrol on the Hh signaling pathway and EMT in gastric cancer in vitro. Gastric cancer SGC-7901 cells were treated with resveratrol or cyclopamine at different concentrations. The viability of the cells was assessed using an MTT assay. The expression of Gli-1, a key component of the Hh signaling pathway, and Snail, E-cadherin and $\mathrm{N}$-cadherin, key components of EMT, was detected by reverse transcription polymerase chain reaction (RT-PCR) and western blotting. The invasion and metastasis of the cells were observed by performing a cell scratch test. The RT-PCR and western blotting showed a decrease in Gli-1, Snail and N-cadherin expression, and an increase in E-cadherin expression in the resveratrol and cyclopamine group compared with the control group, suggesting that resveratrol inhibited the Hh pathway and EMT, as did cyclopamine. The MTT assay indicated that the viability of the SGC-7901 cells was significantly decreased in
\end{abstract}

Correspondence to: Professor Qiong Peng, Department of Gastroenterology, The Third Affiliated Hospital of Anhui Medical University, 390 Huai He Road, Hefei, Anhui 230061, P.R. China E-mail: pengqiong2014@163.com

Key words: hedgehog signaling pathway, resveratrol, cyclopamine, gastric cancer epithelial-mesenchymal transition a concentration-dependent manner following resveratrol and cyclopamine treatment. The cell scratch test showed slower cell invasion and metastasis in the resveratrol and cyclopamine groups. These findings indicated that resveratrol was able to inhibit the Hh signaling pathway and EMT, and suppress invasion and metastasis in gastric cancer in vitro.

\section{Introduction}

Gastric cancer is one of the leading causes of cancer-related mortality in developed and undeveloped countries (1). The five-year survival rate for patients with gastric cancer is $<35 \%$, as the currently available chemotherapeutic regimens are not highly effective. Furthermore, the high recurrence rates and nodal metastasis also require consideration (2).

The process of metastasis is complex and involves the spread of carcinoma cells from the primary site to distant sites for tumor formation. Epithelial-to-mesenchymal transition (EMT) is an essential event in the initial step of the metastatic cascade. The process confers migratory, invasive and stem-like properties upon cells $(3,4)$, and epithelial polarized cells consequently turn into motile mesenchymal-appearing cells (5-7). Cellular phenotype changes have been reported in epithelial cells during EMT, including the loss of cell-cell contacts, cell polarity and epithelial markers, particularly E-cadherin. At the same time, cells acquire mesenchymal markers such as vimentin and $\mathrm{N}$-cadherin (8). Furthermore, cells undergo important changes in their cytoskeleton, which confer mesenchymal phenotypes upon them, such as increased invasiveness and motility $(9,10)$. Blocking or reversing this process is thought to be a promising therapeutic strategy to limit cancer diffusion. EMT is known to be activated by transcriptional regulators, including Snail, Twist and Slug $(6,11)$. The EMT transcription factors have been reported to downregulate the expression of E-cadherin and upregulate the expression of $\mathrm{N}$-cadherin and vimentin $(7,10)$. Moreover, studies have found that Gli-1, a key factor in the Hedgehog (Hh) signaling pathway, can induce the expression of Snail, then decrease the expression of E-cadherin and increase the expression of $\mathrm{N}$-cadherin $(12,13)$. These results suggest that the occurrence of EMT may have a close association with the Hh signaling pathway. 
The Hh signaling pathway is considered to have a vital role in vertebrate development, the homeostatic process and tumorigenesis (14). A previous study reported that Gli-1 is a strong positive activator of downstream target genes and is itself a transcriptional target of the Hh pathway (15), therefore, Gli-1 is regarded as a marker of the abnormal activation of the Hh signaling pathway (16). Recent studies have found that the Hh signaling pathway is abnormally activated in small cell lung, breast, prostate, colorectal and pancreatic cancer (17-21). Significantly, the Sonic Hh (Shh) signaling pathway has been shown to contribute to tumor metastasis by inducing EMT in gastric cancer (22).

Currently, the Hh signaling pathway is a focus of attention as a therapeutic target for anti-cancer treatment. A number of experiments in vivo and in vitro have proved that cyclopamine, a cell-steroidal alkaloid, is specifically antagonistic with the Hh signaling pathway through direct interaction with Smo (23). The drug is a valuable tool that aids in determining the involvement of Hh signaling in the development of a variety of tumors. Cyclopamine is treated as a type of cancer-fighting drug with further promising applications, but its high price reduces its clinical application greatly. Hence, due to this, it is important to search for another anticancer drug.

The natural polyphenolic compound, resveratrol (trans-3,5,4-trihydroxystilbene), is found in peanuts, grapes and a variety of berries, as well as in food products derived from these foods, such as wine $(24,25)$. Resveratrol exhibits a number of beneficial health effects, including anti-inflammatory, anti-oxidant, cardioprotective and anti-tumor properties $(26,27)$, as well as affecting tumorigenesis and tumor progression (28).

In particular, study findings have indicated that resveratrol inhibits EMT in lung, pancreatic and breast cancer (29-31), and that it is also able to suppress the Hh signaling pathway in prostate carcinoma (32). In addition, resveratrol has been identified as being able to prevent, reverse or delay carcinogenesis. Notably, the drug has also been shown to prevent or delay cancer progression, which may be, in part, due to its ability to affect EMT-type cells by the attenuation of the Wnt and Hh signaling pathways (33). However, there have been no studies showing whether resveratrol inhibits the Hh signaling pathway to regulate the EMT in gastric cancer. The aim of the present study was to investigate the potential use of resveratrol as an inhibitor of the Hh pathway, which regulate EMTs, in gastric cancer.

\section{Materials and methods}

Reagents and cell culture. Resveratrol and cyclopamine were purchased from Sigma-Aldrich (St. Louis, MO, USA) and dissolved in dimethylsulfoxide (DMSO; Sigma-Aldrich). Stock solutions $(100 \mathrm{mmol} / 1$ resveratrol and $20 \mathrm{mmol} / 1$ cyclopamine in DMSO) were stored in the dark at $-20^{\circ} \mathrm{C}$ and diluted with RPMI-1640 medium (HyClone, Logan, UT, USA) immediately prior to use. The final concentration of DMSO in the RPMI-1640 medium was maintained at $<0.1 \%$. The rabbit polyclonal antibody against Gli-1 and mouse monoclonal antibody against Snail (dilutions with $0.5 \%$ bovine serum albumin) were purchased from Santa Cruz Biotechnology Inc., (catalogue numbers, SC-20687 and SC-271977; dilutions, 1:1,000; Santa Cruz, CA, USA), and other rat monoclonal E-cadherin, mouse monoclonal
$\mathrm{N}$-cadherin and mouse monoclonal $\beta$-tubulin antibodies (dilutions all with $0.5 \%$ bovine serum albumin) were obtained from Sigma-Aldrich (catalogue numbers, U3254, C2542 and T8328; dilutions, 1:1,000) .

The human gastric cancer SGC-7901 cell line was obtained from The Central Laboratory of the Third Affiliated Hospital of Anhui Medical University (Hefei, Anhui, China). The SGC-7901 cells were grown in RPMI-1640 medium containing $2 \mathrm{mM}$ L-glutamine supplemented with $10 \%$ fetal bovine serum (Gibco BRL, Life Technologies Inc., Grand Island, NY, USA), penicillin $(100 \mathrm{IU} / \mathrm{ml})$ and streptomycin $(100 \mu \mathrm{g} / \mathrm{ml})$ at $37^{\circ} \mathrm{C}$ in a humidified atmosphere with $5 \% \mathrm{CO}_{2}$, and harvested with trypsin-EDTA when the cells had reached exponential growth.

Cell proliferation assay. The MTT assay was used to determine the antitumor effects of the two drugs. Briefly, the cells were seeded at a density of $1 \times 10^{4}$ in 96 -well plates. Following overnight culture, the cells were then treated with increasing doses of resveratrol or cyclopamine for $48 \mathrm{~h}$. The $\mathrm{IC}_{50}$ value was the concentration resulting in $50 \%$ cell growth inhibition by a 48-h exposure to drug compared with the untreated control cells. Following cell exposure to each drug for $48 \mathrm{~h}$ in 96-well plates, $20 \mu \mathrm{l}$ MTT (Sigma-Aldrich; $5 \mathrm{mg} / \mathrm{ml}$ ) solution was added to each well and then the optical density (OD) of each well was determined at $490 \mathrm{~nm}$ on an ELISA plate reader (Bio-Rad Laboratories, Inc., Winooski, VT, USA) following $4 \mathrm{~h}$ of incubation at $37^{\circ} \mathrm{C}$. The percentage of cell growth inhibition resulting from each drug was calculated as: [(OD490 control cells - OD490 $\left.0_{\text {treated cells }}\right) /$ OD490 $\left.0_{\text {control cells }}\right]$ x 100. This assay was repeated in more than three independent experiments.

Cell scratch test. A scratch was made across the monolayer of SGC-7901 cells seeded in 6-well plates. The cells were treated with resveratrol and cyclopamine alone at their respective $\mathrm{IC}_{50}$ values. Images of the scratches were captured at $0,12,24$ and $48 \mathrm{~h}$ using a digital camera (C5060, Olympus, Tokyo, Japan) mounted on an inverted microscope (CKX41, Olympus).

Reverse transcription-polymerase chain reaction (RT-PCR) analysis. Total RNA was prepared from the cells using TRIzol reagent (Invitrogen Life Technologies, Carlsbad, CA, USA), according to the manufacturer's instructions. RT to cDNA was performed in the PCR thermal cycler (S1000; Biorad, California, USA) for $10 \mathrm{~min}$ at $25^{\circ} \mathrm{C}, 70 \mathrm{~min}$ at $37^{\circ} \mathrm{C}$ and $10 \mathrm{~min}$ at $72^{\circ} \mathrm{C}$. Specific primers for the PCR amplification were designed and are listed in Table I. The PCR thermal cycle profile was composed of one cycle of denaturation for $5 \mathrm{~min}$ at $95^{\circ} \mathrm{C}, 30$ cycles of denaturation for $30 \mathrm{sec}$ at $95^{\circ} \mathrm{C}$, annealing of primers for $45 \mathrm{sec}$ at different temperatures, and extension for $50 \mathrm{sec}$ at $72^{\circ} \mathrm{C}$, followed by one cycle of a final extension step at $72^{\circ} \mathrm{C}$ for $10 \mathrm{~min}$. PCR products were assayed by electrophoresis in a $1 \%$ agarose gel using Tris-acetate/ EDTA buffer and visualized by $0.01 \%$ ethidium bromide. Relative expression was determined by densitometry using ImageJ software (Version 1.36b; NIH, Bethesda, MD, USA). The mean values were normalized to the internal GAPDH control and were calculated from at least three independent experiments. 
Table I. Primer sequences for reverse transcription-poymerase chain reaction assays.

\begin{tabular}{ll}
\hline Genes & \multicolumn{1}{c}{ Sequences } \\
\hline Gli-1 & \\
Forward & P'-TTCCTACCAG, bp \\
Reverse & 5'-CCCTATGTGAAGCCCTATTT-3' \\
Snail & \\
Forward & 5'-CCTGGGTGCCCTCAAGATGC-3' \\
Reverse & 5'-GAGCAGGGACATTCGGGAGA-3' \\
N-cad & \\
Forward & 5'-CGTGAAGGTTTGCCAGTGTG-3' \\
Reverse & 5'-GCGTTCTTTATCCCGCCGTT-3' \\
E-cad & \\
Forward & 5'-CCTCAGGTCATAAACATCATTG-3' \\
Reverse & 5'-CGCCTCCTTCTTCATCATAGTAA-3' \\
GAPDH & \\
Forward & 5'-CAAGGTCATCCATGACAACTTCG-3' \\
Reverse & 5'-GTCCACCACCCTGTTGCTGTAG-3' \\
\hline
\end{tabular}

N-cad, N-cadherin; E-cad, E-cadherin.
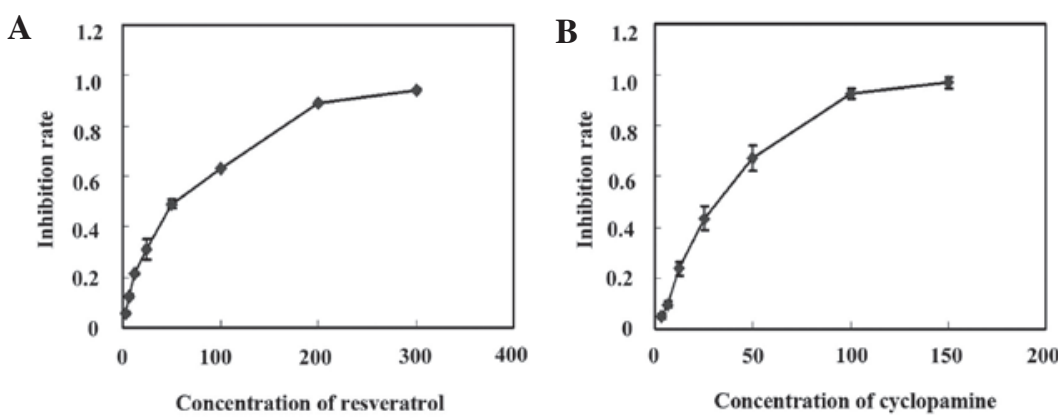

Figure 1. MTT assays were used to examine the inhibitory effect of resveratrol and cyclopamine on cell proliferation. The SGC-7901 cells were exposed to varying concentrations of (A) resveratrol (3.125-300 $\mu \mathrm{mol} / 1)$ or (B) cyclopamine (3.125-150 $\mu \mathrm{mol} / \mathrm{l})$. Each data point is the result of more than three independent experiments.

Western blot analysis. The SGC-7901 cells $\left(1 \times 10^{6} /\right.$ well) were cultured on $100-\mathrm{mm}^{2}$ plates overnight and treated with resveratrol or cyclopamine for $48 \mathrm{~h}$, at their respective $\mathrm{IC}_{50}$ levels. The cells were washed with ice-cold phosphate-buffered saline (PBS) and scraped in lysis buffer. The lysates were centrifuged at $13,380 \mathrm{x}$ g for $30 \mathrm{~min}$ at $4^{\circ} \mathrm{C}$ and the supernatant was collected. Equivalent cell proteins were analyzed by sodium dodecyl sulfate-polyacrylamide gel electrophoresis and transferred to polyvinylidene difluoride membranes. The membranes were blocked in PBS containing 5\% skimmed milk and $0.1 \%$ Tween- 20 for $2 \mathrm{~h}$. Next, the appropriate primary antibodies against Gli-1, Snail, E-cadherin, N-cadherin and $\beta$-tubulin were used and incubated overnight at $4^{\circ} \mathrm{C}$. The visualization of proteins was performed with a horseradish peroxidase-conjugated goat anti-rabbit, goat anti-mouse and goat anti-rat secondary antibodies (catalogue numbers, ZB-2301, ZB-2305 and ZB-2307, respectively; dilution, 1:5,000; ZSGB-BIO, Beijing, China) at room temperature for $1 \mathrm{~h}$, and the protein bands were detected using enhanced chemiluminescence reagents (Millipore, Billerica, MA, USA).
Statistical analysis. The results obtained from at least three independent experiments are expressed as the mean \pm standard deviation. Student's t-test was used to determine the differences between the control and treatment groups. $\mathrm{P}<0.05$ was considered to indicate a statistically significant difference.

\section{Results}

Different antiproliferative effects of resveratrol and cyclopamine in SGC-7901 cells. The effects of resveratrol and cyclopamine on the proliferation of the SGC-7901 cells were determined using an MTT assay. The dose-dependent growth inhibitory effects of resveratrol or cyclopamine were observed in the SGC-7901 cells (Fig. 1). The $\mathrm{IC}_{50}$ values of resveratrol and cyclopamine were $54.92 \pm 2.52 \mu \mathrm{M}$ and $26.14 \pm 1.09 \mu \mathrm{M}$.

Resveratrol and cyclopamine inhibits the metastasis and invasion of SGC-7901 cells. The width of the scratch was measured under a microscope at $0,12,24$ and $48 \mathrm{~h}$. The results (Fig. 2) showed that the scratches of the cells treated 


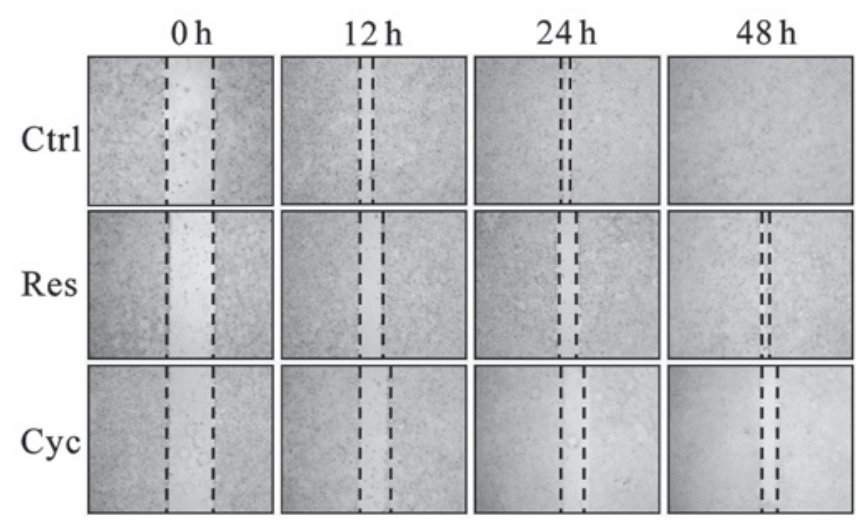

Figure 2. Effect of resveratrol and cyclopamine on the metastasis and invasion of SGC-7901 cells. The cells were cultured with RPMI-1640 medium (Ctrl), resveratrol (Res) and cyclopamine (Cyc) at their respective $\mathrm{IC}_{50}$ values for $48 \mathrm{~h}$. Scratches of the same width were made for each group at $0 \mathrm{~h}$, and then the width of the scratched was measured at 12,24 and $48 \mathrm{~h}$.

A

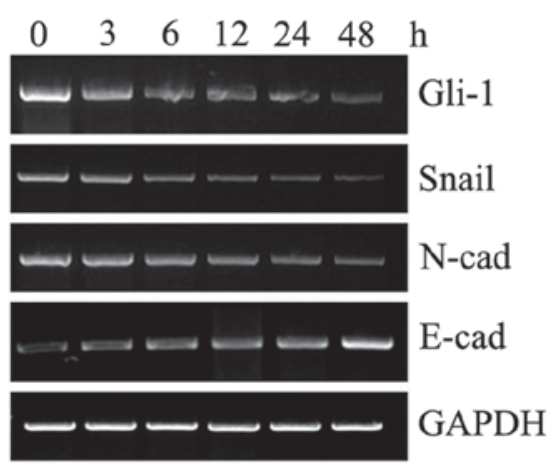

B

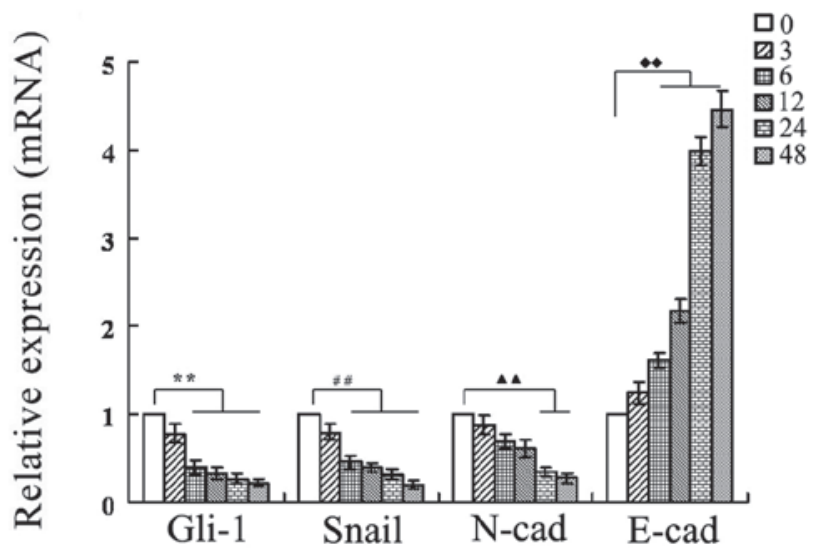

Figure 3. Various effects of resveratrol exposure $\left(\mathrm{IC}_{50}\right.$ value) on the target genes for different times. The SGC-7901 cells were cultured with resveratrol $(55 \mu \mathrm{mol} / 1)$ for $0,3,6,12,24$ and $48 \mathrm{~h}$. (A) The expression levels of the mRNA of Gli-1, Snail, E-cadherin and N-cadherin were detected by reverse transcription-polymerase chain reaction assay. GAPDH was used as a loading control. (B) The relative mRNA expression of the target genes. ${ }^{* *} \mathrm{P}<0.01 \mathrm{vs}$. Gli-1 0 h; ${ }^{\# \#} \mathrm{P}<0.01$ vs. Snail 0 h; ${ }^{\mathbf{\Delta}} \mathrm{P}<0.01$ vs. N-cadherin 0 h; ${ }^{* *} \mathrm{P}<0.01$ vs. E-cadherin 0 h. E-cad, E-cadherin; $\mathrm{N}$-cad, N-cadherin.

with resveratrol or cyclopamine were wider than the control group at the same time-points. This indicated that resveratrol and cyclopamine were able to inhibit metastasis and invasion in the human gastric cancer SGC-7901 cell line.

Different effects of resveratrol on the expression of Gli-1, Snail, E-cadherin and N-cadherin at different time-points. In order to determine the action time of resveratrol for inhibiting the Hh signaling pathway and EMT, the SGC-7901 cells were cultured in RPMI-1640 medium containing $55 \mu \mathrm{mol} / 1$ resveratrol for $0,3,6,12,24$ and $48 \mathrm{~h}$ respectively, and then the expression of Gli-1, Snail, E-cadherin and N-cadherin was analyzed using RT-PCR and western blotting. The results (Figs. 3 and 4) showed that the expression of Gli-1, Snail and $\mathrm{N}$-cadherin reduced, while the expression of E-cadherin increased, in a time-dependent manner. In particular, the results indicated that the changes in E-cadherin expression were more marked at $24 \mathrm{~h}$, therefore, the study used $24 \mathrm{~h}$ as the action time of resveratrol in the gastric cancer SGC-7901 cells.

Changes in the expression of Gli-1, Snail, E-cadherin and $N$-cadherin mRNA following treatment of cells with resveratrol or cyclopamine for $24 \mathrm{~h}$. To confirm the ability of resveratrol to inhibit the Hh signaling pathway and EMT, SGC-7901 cells treated with complete medium (Ctrl group), $55 \mu \mathrm{mol} / 1$ resveratrol (Res group) or $25 \mu \mathrm{mol} / 1$ cyclopamine (Cyc group) for $24 \mathrm{~h}$ were used to examine changes in the Hh signaling pathway and in EMT markers. As shown in Fig. 5, the results indicated that the expression of E-cadherin was significantly increased, while that of Gli-1, Snail and $\mathrm{N}$-cadherin was significantly in the Res and Cyc groups compared with the Ctrl group. In other words, resveratrol inhibited Gli-1 expression, and then downregulated Snail and $\mathrm{N}$-cadherin expression, and upregulated E-cadherin expression, which was identical to cyclopamine with regard to mRNA expression.

Expression of Gli-1, Snail, E-cadherin and N-cadherin protein following treatment of cells with resveratrol or cyclopamine for $24 \mathrm{~h}$. In order to further demonstrate the effect of resveratrol on Gli-1, Snail, E-cadherin and N-cadherin from the perspective of protein expression, the western blotting experiment was used to analyze Gli-1, Snail, E-cadherin and $\mathrm{N}$-cadherin protein expression in the $\mathrm{Ctrl}$, Res and Cyc groups. The results (Fig. 6) showed that the expression of E-cadherin was markedly enhanced in the Res and Cyc 
A

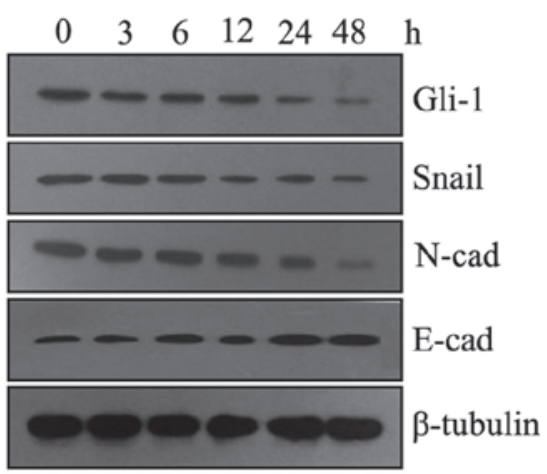

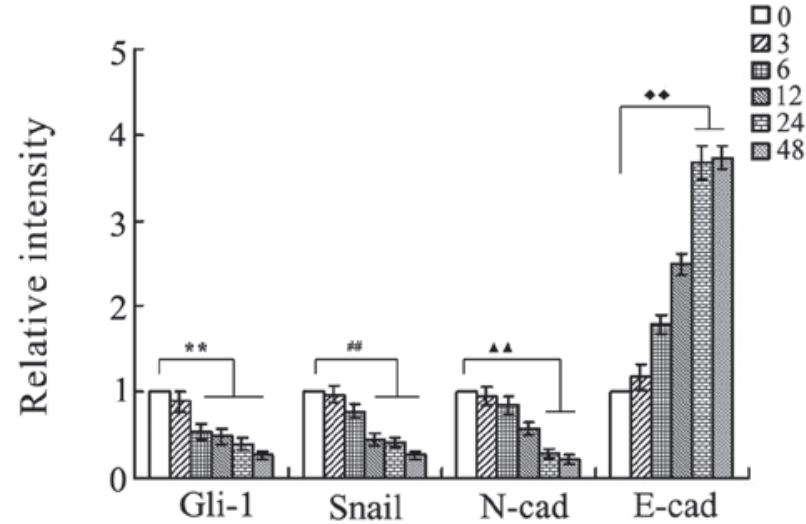

Figure 4. Different effects of resveratrol exposure for different times at its $\mathrm{IC}_{50}$ concentration. (A and B) The human gastric cancer SGC-7901 cells were treated in complete medium containing resveratrol (55 $\mu \mathrm{mol} / \mathrm{l})$ for $0,3,6,12,24$ and $48 \mathrm{~h}$. Subsequent to treatment, the proteins of Gli-1 and EMT markers were analyzed by western blot analysis with corresponding antibodies. $\beta$-tubulin was used as a loading control. ${ }^{* *} \mathrm{P}<0.01 \mathrm{vs}$. Gli-1 0 h; ${ }^{\# \#} \mathrm{P}<0.01 \mathrm{vs}$. Snail 0 h; ${ }^{\boldsymbol{\Lambda}} \mathrm{P}<0.01$ vs. $\mathrm{N}$-cadherin $0 \mathrm{~h} ;{ }^{\bullet} \mathrm{P}<0.01$ vs. E-cadherin 0 h. E-cad, E-cadherin; $\mathrm{N}$-cad, $\mathrm{N}$-cadherin.

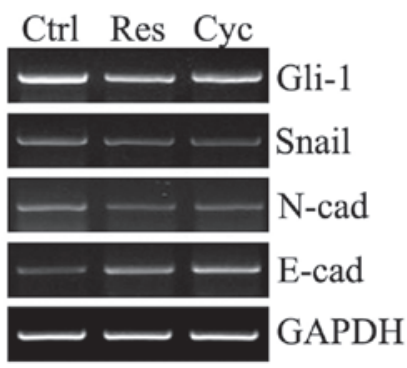

B

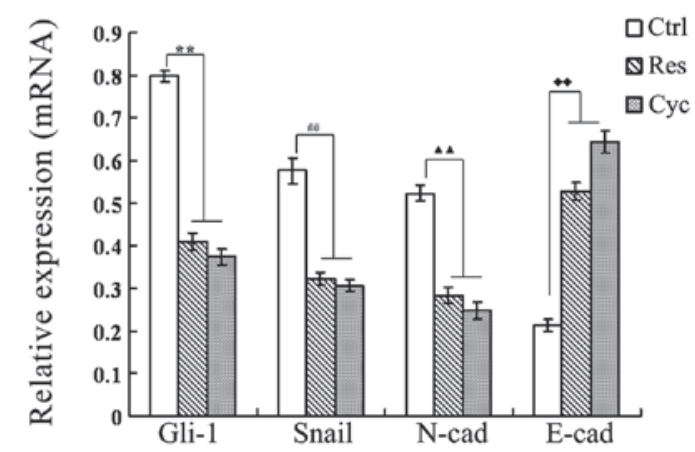

Figure 5. (A and B) Expression of Gli-1, Snail, E-cadherin and N-cadherin mRNA in untreated (Ctrl), resveratrol-treated (55 $\mu \mathrm{mol} / 1$; Res) and cyclopamine-treated ( $25 \mu \mathrm{mol} / 1$; Cyc) SGC-7901 cells was assessed by reverse transcription-polymerase chain reaction analysis. The expression of each gene was standardized using GAPDH as a reference gene. The relative intensity for each band was measured using ImageJ software, dividing the absolute intensity of each sample band by the absolute intensity of the standard. ${ }^{* *} \mathrm{P}<0.01$ vs. control (Gli-1); ${ }^{\# \#} \mathrm{P}<0.01$ vs. control (Snail); ${ }^{\boldsymbol{\Delta} \mathbf{\Lambda}} \mathrm{P}<0.01 \mathrm{vs}$. control (N-cadherin); "• $\mathrm{P}<0.01$ vs. control (E-cadherin). E-cad, E-cadherin; $\mathrm{N}$-cad, N-cadherin.

A

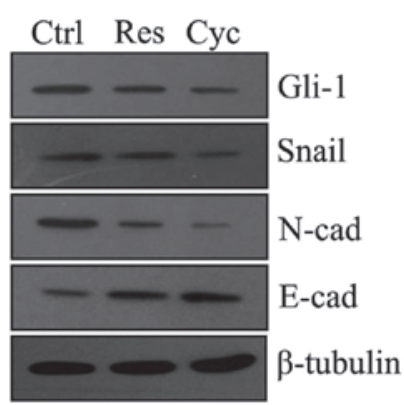

B

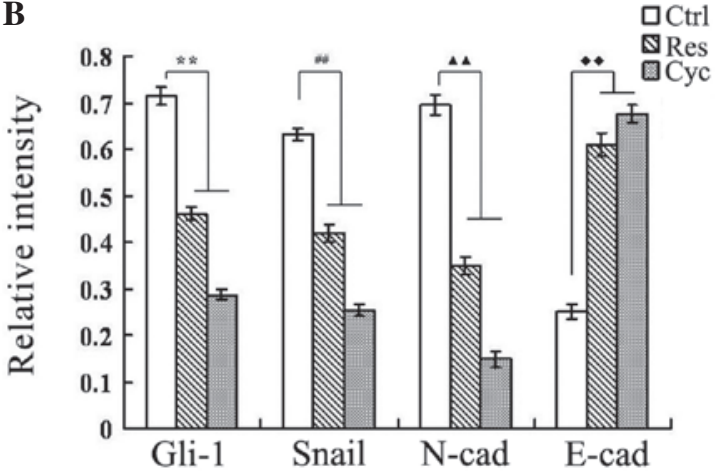

Figure 6. Proteins expression level of Gli-1, Snail, E-cadherin and N-cadherin in untreated (Ctrl), resveratrol-treated (Res) and cyclopamine-treated (Cyc) SGC-7901 cells with respective IC $_{50}$ values, assessed by western blotting analysis. Proteins were extracted with radioimmunoprecipitation assay buffer. Specific antibodies against Gli-1, Snail, E-cadherin and N-cadherin were used. Bound proteins were detected using appropriate horseradish peroxidase-conjugated secondary antibodies. (A) Analysis of the western blot images was performed by calculating (B) the relative intensity of the immunoreactive bands following analysis by means of the ImageJ software. The relative densities of the bands were expressed as arbitrary units. ${ }^{* *} \mathrm{P}<0.01$ vs. control (Gli-1); ${ }^{\# \#} \mathrm{P}<0.01 \mathrm{vs}$. control

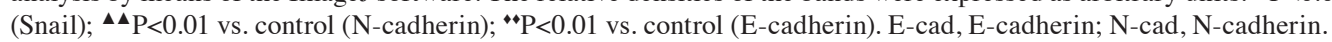

groups compared with the Ctrl group, while at the same time, Gli-1, Snail and N-cadherin protein expression was decreased significantly in the Res and Cyc groups compared with the Ctrl group.

\section{Discussion}

Resveratrol is a natural polyphenolic compound that was first isolated from the roots of the white hellebore (Veratrun album), 
and is now known to be found in mulberries, grapes, red wine, peanuts, knotweed and other plants $(27,34)$. Resveratrol is a member of the phytoalexin family, which are low molecular weight, secondary metabolites produced by plants as a defensive mechanism against certain stresses, such as attack by pathogens, wounds and ultraviolet irradiation. Numerous studies in the literature have indicated that resveratrol exhibits anticancer, anti-inflammatory, anti-oxidative and antiasthmatic effects $(26,27,35,36)$, suppresses metastasis in several cancers (37), inhibits platelet aggregation in cardiovascular diseases, and regulates mucin, inflammatory mediators [monocyte chemoattractant protein-1, interleukin (IL)-6 and IL-8] and matrix-metalloprotease-9 expression in chronic obstructive pulmonary disease (38-40). Another study revealed that resveratrol inhibits the Hh signaling pathway in prostate cancer (32). These beneficial properties mean that resveratrol can be used as a potential health-benefiting compound in the manufacture of pharmaceuticals, cosmetics and nutraceuticals.

The $\mathrm{Hh}$ gene was initially identified by Christiane Nüsslein-Volhard and Eric Wieschaus in 1980, while studying mutations of the fruit fly, Drosophila melanogaster, which directly affected embryonic development (41). There are three Hh genes in vertebrates: Shh, Desert Hh (Dhh) and Indian Hh (Ihh), each of which were independently identified (42-45). The corresponding hh proteins of humans and mice are secreted and membrane anchored, and can act on nearby and distant cells (46). The Hh signaling pathway is composed of Patched (Ptch), a 12-transmembrane receptor, Smoothened (Smo), a 7-transmembrane receptor, and the Glis transcription factor family (Gli-1, Gli-2 and Gli-3) (47). The Hh signaling pathway in mammalian cells is mediated by ligands of $\mathrm{Hh}$. In the absence of Hh, the activity of Smo is inhibited by Ptch, resulting $\mathrm{Hh}$ signaling pathway inactivation. Binding of $\mathrm{Hh}$ to Ptch abrogates its inhibitory effect, and the repression of Smo is therefore halted, consequently activating the Glis transcription factor family $(48,49)$. Gli-1 is a strong positive activator of downstream target genes and is itself a transcriptional target of the Hh pathway in the Glis transcription factor family (15), however, Gli-2 has the double function of activation and inhibition, and the main effect of Gli-3 is inhibition. As a consequence, Gli-1 is regarded as a marker of the abnormal activation of the Hh signaling pathway (16). In addition, a recent study has indicated that the Shh signaling pathway promotes metastasis via activation of the EMT pathway in gastric cancer (22).

EMT plays a role in cancer invasion and metastasis, which are key processes in cancer progression and metastasis. Zinc-finger transcription factors, such as Snail, Twist1 and Slug, trigger the EMT process and repress E-cadherin transcription. These transcriptional repressors cadherin also aid in the development of EMT (6). In the process of EMT, the characteristic marker, E-cadherin, is lost by the epithelial cells and the mesenchymal marker, N-cadherin, is gained. Following the occurrence of EMT, the solid connection between the cells and the cell polarity are destroyed, and intercellular adhesion is weakened or lost, so the carcinoma cells are prone to invasion and metastasis. A previous study has shown that Gli-1, the Hh signaling pathway target gene, can rapidly and directly induce the expression of EMT transcription factor Snail (50), thereby downregulating the expression of E-cadherin and upregulate the expression of $\mathrm{N}$-cadherin. This indicates that the EMT process is regulated by the Hh signaling pathway. Another previous study demonstrated that resveratrol could inhibit EMT in order to slow migration and invasion in pancreatic cancer cells (51).

However, there have been no studies demonstrating that resveratrol can inhibit the Hh signaling pathway and EMT in human gastric cancer cells. Therefore, the present study aimed to prove that resveratrol suppresses the Hh signaling pathway and further inhibits the EMT process, finally inhibiting invasion and metastasis in gastric cancer.

In the present study, cyclopamine was used as a positive control, as it is the classical inhibitor of the Hh signaling pathway. Cyclopamine is a natural steroidal alkaloid derived from the corn lily, Veratrum californicum, which specifically disrupts the Hh signaling pathway (52). Using a mechanism similar to that identified for the Hh-induced pathway activation and for small-molecule Hh agonists, cyclopamine promotes the ciliary translocation of Smo (53). In addition, cyclopamine is believed to result in a conformational shift of Smo that renders the receptor inactive and effectively blocks further signal transduction $(54,55)$.

In the present study, when the SGC-7901 cells were treated with resveratrol or cyclopamine, SGC-7901 cell invasion and metastasis was suppressed (Fig. 2). The results in Figs. 5 and 6 show that the expression of Gli-1 was inhibited by resveratrol and cyclopamine, and that the expression of Snail and N-cadherin was reduced. However, the expression of E-cadherin was increased. These results may be explained by the fact that the EMT-inducing transcription factor, Snail, is induced by Gli-1 directly (50), therefore, the expression of Snail is reduced when Gli-1 is restrained. Snail plays a central role in EMT, as it can bind to the promoter E-box, which represses E-cadherin transcription (6). Therefore the expression of E-cadherin increases when Snail is inhibited.

In conclusion, the present study demonstrated that resveratrol is able to inhibit gastric cancer cell invasion and metastasis in vitro by inhibiting the Hh signaling pathway and EMT. However, confirmation of this result in a larger number of gastric cancer cell lines is necessary and further in vivo studies are required.

\section{Acknowledgements}

This study was supported by the Central Laboratory of the Third Affiliated Hospital of Anhui Medical University. The authors would like to thank Miss. Yuan Yuan for providing technical assistance.

\section{References}

1. Parkin DM: Global cancer statistics in the year 2000. Lancet Oncol 2: 533-543, 2001.

2. Khosravi Shahi P, Díaz Muñoz de la Espada VM, García Alfonso P, et al: Management of gastric adenocarcinoma. Clin Transl Oncol 9: 438-442,2007.

3. Radisky DC: Epithelial-mesenchymal transition. J Cell Sci 118: 4325-4326, 2005.

4. Thiery JP and Sleeman JP: Complex networks orchestrate epithelial-mesenchymal transitions. Nat Rev Mol Cell Biol 7: 131-142, 2006.

5. Chang CJ, Chao CH, Xia W, et al: p53 regulates epithelial-mesenchymal transition and stem cell properties through modulating miRNAs. Nat Cell Biol 13: 317-323, 2011.

6. Thiery JP, Acloque H, Huang RY and Nieto MA: Epithelial-mesenchymal transitions in development and disease. Cell 139: 871-890, 2009. 
7. Voulgari A and Pintzas A: Epithelial-mesenchymal transition in cancer metastasis: mechanisms markers and strategies to overcome drug resistance in the clinic. Biochim Biophys Acta 1796: 75-90, 2009

8. Kundu JK and Surh YJ: Cancer chemopreventive and therapeutic potential of resveratrol: mechanistic perspectives. Cancer Lett 269: 243-261, 2008.

9. Xie D, Gore C, Liu J, et al: Role of DAB2IP in modulating epithelial-to-mesenchymal transition and prostate cancer metastasis. Proc Natl Acad Sci USA 107: 2485-2490, 2010.

10. Gjerdrum C, Tiron C, Høiby T, et al: Axl is an essentia epithelial-to-mesenchymal transition-induced regulator of breast cancer metastasis and patient survival. Proc Natl Acad Sci USA 107: 1124-1129, 2010

11. Moreno-Bueno G, Peinado H, Molina P, et al: The morphological and molecular features of the epithelial-to-mesenchymal transition. Nat Protoc 4: 1591-1613, 2009.

12. Li X, Deng W, Nail CD, Bailey SK, Kraus MH, Ruppert JM and Lobo-Ruppert SM: Snail induction is an early response to Gli1 that determines the efficiency of epithelial transformation. Oncogene 25: 609-621, 2006.

13. Louro ID, Bailey EC, Li X, et al: Comparative gene expression profile analysis of GLI and c-MYC in an epithelial model of malignant transformation. Cancer Res 62: 5867-5873, 2002.

14. McMahon AP, Ingham PW and Tabin CJ: Developmental roles and clinical significance of hedgehog signaling. Curr Top Dev Biol 53: 1-114, 2003.

15. Lee J, Platt KA, Censullo P and Ruiz i Altaba A: Gli1 is a target of Sonic hedgehog that induces ventral neural tube development. Development 124: 2537-2552, 1997.

16. Ohta M, Tateishi K, Kanai F, et al: p53-independent negative regulation of $\mathrm{p} 21 /$ cyclin-dependent kinase-interacting protein 1 by the sonic hedgehog-glioma-associated oncogene 1 pathway in gastric carcinoma cells. Cancer Res 65: 10822-10829, 2005.

17. Watkins DN, Berman DM, Burkholder SG, Wang B, Beachy PA and Baylin SB: Hedgehog signalling within airway epithelial progenitors and in small-cell lung cancer. Nature 422: 313-317, 2003

18. Kubo M, Nakamura M, Tasaki A, et al: Hedgehog signaling pathway is a new therapeutic target for patients with breast cancer. Cancer Res 64: 6071-6074, 2004

19. Mimeault M, Moore E, Moniaux N, et al: Cytotoxic effects induced by a combination of cyclopamine and gefitinib, the selective hedgehog and epidermal growth factor receptor signaling inhibitors, in prostate cancer cells. Int J Cancer 118: 1022-1031, 2006.

20. Qualtrough D, Buda A, Gaffield W, Williams AC and Paraskeva C: Hedgehog signalling in colorectal tumour cells: induction of apoptosis with cyclopamine treatment. Int J Cancer 110: 831-837, 2004.

21. Thayer SP, di Magliano MP, Heiser PW, et al: Hedgehog is an early and late mediator of pancreatic cancer tumorigenesis. Nature 425: 851-856, 2003.

22. Yoo YA, Kang MH, Lee HJ, et al: Sonic hedgehog pathway promotes metastasis and lymphangiogenesis via activation of Akt, EMT and MMP-9 pathway in gastric cancer. Cancer Res 71: 7061-7070, 2011

23. Yanai K, Nagai S, Wada J, et al: Hedgehog signaling pathway is a possible therapeutic target for gastric cancer. J Surg Oncol 95: 55-62, 2007.

24. Wang Y, Catana F, Yang Y, Roderick R and van Breemen RB An LC-MS method for analyzing total resveratrol in grape juice, cranberry juice and in wine. J Agric Food Chem 50 431-435, 2002

25. Burns J, Yokota T, Ashihara H, Lean ME and Crozier A: Plant foods and herbal sources of resveratrol. J Agric Food Chem 50: 3337-3340, 2002

26. Qadri SM, Föller M and Lang F: Inhibition of suicidal erythrocyte death by resveratrol. Life Sci 85: 33-38, 2009.

27. Baur JA and Sinclair DA: Therapeutic potential of resveratrol: the in vivo evidence. Nat Rev Drug Discov 5: 493-506, 2006.

28. Kundu JK and Surh YJ: Cancer chemopreventive and therapeutic potential of resveratrol: mechanistic perspectives. Cancer Lett 269: 243-261, 2008.

29. Wang H, Zhang H, Tang L, et al: Resveratrol inhibits TGF- $\beta 1$-induced epithelial-to-mesenchymal transition and suppresses lung cancer invasion and metastasis. Toxicology 303: 139-146, 2013

30. Li W, Ma J, Ma Q, et al: Resveratrol inhibits the epithelial-mesenchymal transition of pancreatic cancer cells via suppression of the PI-3 K/Akt/NF-kB pathway. Curr Med Chem 20: $4185-4194,2013$
31. Vergara D, Valente CM, Tinelli A, et al: Resveratrol inhibits the epidermal growth factor-induced epithelial mesenchymal transition in MCF-7 cells. Cancer Lett 310: 1-8, 2011.

32. Slusarz A, Shenouda NS, Sakla MS, et al: Common botanical compounds inhibit the hedgehog signaling pathway in prostate cancer. Cancer Res 70: 3382-3390, 2010.

33. Sarkar FH, Li Y, Wang Z and Kong D: The role of nutraceuticals in the regulation of Wnt and Hedgehog signaling in cancer. Cancer Metastasis Rev 29: 383-394, 2010.

34. Shakibaei M, Harikumar KB and Aggarwal BB: Resveratrol addiction: to die or not to die. Mol Nutr Food Res 53: 115-128, 2009.

35. Bisht K, Wagner $\mathrm{KH}$ and Bulmer AC: Curcumin, resveratrol and flavonoids as anti-inflammatory, cyto- and DNA-protective dietary compounds. Toxicology 278: 88-100, 2010.

36. Michels G, Wätjen W, Weber N, et al: Resveratrol induces apoptotic cell death in rat H4IIE hepatoma cells but necrosis in C6 glioma cells. Toxicology 225: 173-182, 2006.

37. Busquets S, Ametller E, Fuster G, et al: Resveratrol a natural diphenol, reduces metastatic growth in an experimental cancer model. Cancer Lett 245: 144-148, 2007.

38. Olas B, Wachowicz B, Tomczak A, et al: Comparative anti-platelet and antioxidant properties of polyphenol-rich extracts from: berries of Aronia melanocarpa, seeds of grape and bark of Yucca schidigera in vitro. Platelets 19: 70-77, 2008.

39. Knobloch J, Hag H, Jungck D, Urban K and Koch A: Resveratrol impairs the release of steroid-resistant cytokines from bacterial endotoxin-exposed alveolar macrophages in chronic obstructive pulmonary disease. Basic Clin Pharmacol Toxicol 109: 138-143, 2011.

40. Lee SY, Lee HJ, Sikder MA, et al: Resveratrol inhibits mucin gene expression production and secretion from airway epithelial cells. Phytother Res 26: 1082-1087, 2012.

41. Nüsslein-Volhard C and Wieschaus E: Mutations affecting segment number and polarity in Drosophila. Nature 287: 795-801, 1980.

42. Chang DT, López A, von Kessler DP, et al: Products, genetic linkage and limb patterning activity of a murine hedgehog gene. Development 120: 3339-3353, 1994.

43. Echelard Y, Epstein DJ, St-Jacques B, et al: Sonic hedgehog, a member of a family of putative signaling molecules, is implicated in the regulation of CNS polarity. Cell 75: 1417-1430, 1993.

44. Krauss S, Concordet JP and Ingham PW: A functionally conserved homolog of the Drosophila segment polarity gene $\mathrm{hh}$ is expressed in tissues with polarizing activity in zebrafish embryos. Cell 75: 1431-1444, 1993.

45. Riddle RD, Johnson RL, Laufer E and Tabin C: Sonic hedgehog mediates the polarizing activity of the ZPA. Cell 75: 1401-1416, 1993.

46. Lum L and Beachy PA: The hedgehog response network: sensors, switches and routers. Science 304: 1755-1759, 2004.

47. Eaton S: Multiple roles for lipids in the Hedgehog signalling pathway. Nat Rev Mol Cell Biol 9: 437-445, 2008.

48. Pasca di Magliano $M$ and Hebrok M: Hedgehog signalling in cancer formation and maintenance. Nat Rev Cancer 3: 903-911, 2003

49. Ingham PW and McMahon AP: Hedgehog signaling in animal development: paradigms and principles. Genes Dev 15: 3059-3087, 2001.

50. Louro ID, Bailey EC, Li X, et al: Comparative gene expression profile analysis of GLI1 and c-MYC in an epithelial model of malignant transformation. Cancer Res 62: 5867-5873, 2002

51. Shankar S, Nall D, Tang SN, Meeker D, Passarini J, Sharma J and Srivastava RK: Resveratrol inhibits pancreatic cancer stem cell characteristics in human and KrasG12D transgenic mice by inhibiting pluripotency maintaining factors and epithelial-mesenchymal transition. PLoS One 6: e16530, 2011.

52. Incardona JP, Gaffield W, Kapur RP and Roelink H: The teratogenic Veratrum alkaloid cyclopa mine inhibits Sonic hedgehog signal transduction. Development 125: 3553-3562, 1998.

53. Wang Y, Zhou Z, Walsh CT and McMahon AP: Selective translocation of intracellular Smoothened to the primary cilium in response to Hedgehog pathway modulation. Proc Natl Acad Sci USA 106: 2623-2628, 2009

54. Chen JK, Taipale J, Cooper MK and Beachy PA: Inhibition of Hedgehog signaling by direct binding of cyclopamine to Smoothened. Genes Dev 16: 2743-2748, 2002.

55. Scales SJ and de Sauvage FJ: Mechanisms of Hedgehog pathway activation in cancer and implications for therapy. Trends Pharmacol Sci 30: 303-312, 2009. 